BNL-77311-2006-CP

\title{
Single-Transverse Spin Asymmetries
}

\author{
Werner Vogelsang
}

Presented at SPIN 2006

Kyoto, Japan

October 2-7, 2006

\author{
Physics Department \\ Nuclear Theory Group \\ Brookhaven National Laboratory \\ P.O. Box 5000 \\ Upton, NY 11973-5000 \\ WWw.bnl.gov
}

Notice: This manuscript has been authored by employees of Brookhaven Science Associates, LLC under Contract No. DE-AC0298CH10886 with the U.S. Department of Energy. The publisher by accepting the manuscript for publication acknowledges that the United States Government retains a non-exclusive, paid-up, irrevocable, world-wide license to publish or reproduce the published form of this manuscript, or allow others to do so, for United States Government purposes.

This preprint is intended for publication in a journal or proceedings. Since changes may be made before pubtication, it may not be cited or reproduced without the author's permission. 


\section{DISCLAIMER}

This report was prepared as an account of work sponsored by an agency of the United States Government. Neither the United States Government nor any agency thereof, nor any of their employees, nor any of their contractors, subcontractors, or their employees, makes any warranty, express or implied, or assumes any legal liability or responsibility for the accuracy, completeness, or any third party's use or the results of such use of any information, apparatus, product, or process disclosed, or represents that its use would not infringe privately owned rights. Reference herein to any specific commercial product, process, or service by trade name, trademark, manufacturer, or otherwise, does not necessarily constitute or imply its endorsement, recommendation, or favoring by the United States Government or any agency thereof or its contractors or subcontractors. The views and opinions of authors expressed herein do not necessarily state or reflect those of the United States Government or any agency thereof. 


\title{
Single-Transverse Spin Asymmetries
}

\author{
Werner Vogelsang \\ Physics Department, Brookhaven National Laboratory, Upton, New York 11973, U.S.A.
}

\begin{abstract}
We give a brief overview of some of the recent results on single-transverse spin asymmetries, highlighting in particular progress in theoretical understanding.
\end{abstract}

\section{INTRODUCTION}

Single-transverse spin asymmetries (STSAs) play an important role for our understanding of QCD and of nucleon structure. They have a long history, starting from the 1970s and 1980s when surprisingly large STSAs were observed in hadronic reactions like $p^{\uparrow} p \rightarrow \pi X$ at forward angles of the produced pion [1]. The last few years have seen a renaissance in the experimental studies of STSAs. STSAs were investigated in semiinclusive hadron production $e N^{\uparrow} \rightarrow e \pi X$ in deep-inelastic scattering [2], and for proton targets remarkably large asymmetries were found. The advent of the first polarized proton-proton collider, RHIC, has opened new possibilities for extending the studies of STSAs in hadronic scattering into a regime where the use of QCD perturbation theory in the analysis of the data appears to be justified. The STAR [3], PHENIX [4] and BRAHMS [5] collaborations have presented data for the STSA in single-inclusive hadron production. Large effects at forward rapidities persist to RHIC energies.

The observed large size of STSAs has presented a challenge for QCD theorists. Two mechanisms were proposed $[6,7,8]$ and extensively applied $[8,9,10,11]$ in phenomenological studies. The first relies on the use of transverse-momentum dependent (TMD) parton distributions for the transversely polarized proton. For these distributions, known as Sivers functions [6], the parton transverse momentum is assumed to be correlated with the proton spin vector, so that spin asymmetries naturally arise from the directional preference expressed by that correlation. The other mechanism (referred to as EfremovTeryaev-Qiu-Sterman (ETQS) mechanism) is formulated in terms of the collinear factorization approach and twist-three transverse-spin-dependent quark-gluon correlation functions of the proton $[7,8]$. The question of which mechanism should be used in the analysis of a given single-spin asymmetry is primarily tied to the factorization theorem that applies. For the single-inclusive process $p^{\uparrow} p \rightarrow \pi X$, there is only one hard scale, the transverse momentum $\ell_{\perp}$ of the produced pion, and the STSA is power-suppressed ("higher-twist") by $1 / \ell_{\perp}$. In this case, the ETQS mechanism applies [8]. On the other hand, the observables typically investigated in deep-inelastic lepton scattering are characterized by a large scale $Q$ (the virtuality of the DIS photon) and by the much smaller, and also measured, transverse momentum $q_{\perp}$ of the produced hadron. In this two-scale case, single-spin asymmetries may arise at leading twist, i.e., not suppressed by $1 / Q$. The relevant factorization theorem is formulated in terms of the TMD functions $[12,13]$, 
in particular the Sivers functions. Much progress has been made recently in understanding the underlying theoretical issues in the TMD QCD factorization. For example, the gauge-invariance properties of the necessary TMD parton distributions have been clarified $[14,15]$ for DIS and Drell-Yan processes, and an extension to more complicated hadronic processes has been investigated in [16].

In the following, we will describe both types of observables, the single-spin asymmetry in single-inclusive scattering and the asymmetry in a "two-scale" situation. We will also discuss recent work [17] that has connected the two mechanisms in the case of the single-spin asymmetry for the Drell-Yan process.

\section{STSA IN HIGH- $\ell_{\perp}$ PION PRODUCTION IN $p p$ COLLISIONS}

The STSA in the process $p p \rightarrow \pi X$ is among the simplest spin observables in hadronic scattering. One scatters a beam of transversely polarized protons off unpolarized protons and measures the numbers of pions produced to either the left or the right of the plane spanned by the momentum and spin direction of the initial polarized protons. As we mentioned above, measurements of such asymmetries in hadronic scattering have shown spectacular results $[1,3,4,5]$. Large asymmetries of up to several tens of per cents were observed at forward (with respect to the polarized initial beam) angles of the produced pion. Until a few years ago, all these experiments were done with a polarized beam impeding on a fixed target. These experiments necessarily had a relatively limited kinematic reach, in particular in $\ell_{\perp}$. Now, with RHIC, it has become possible to investigate $A_{N}$ at higher energies, in a kinematic regime where the theoretical description has been demonstrated to be under good control $[3,4,5]$.

Power-suppressed contributions to hard-scattering processes are generally much harder to describe in QCD than leading-twist ones. In the case of the single-spin asymmetry in $p p \rightarrow \pi X$, a complete and consistent framework could be developed, however [8]. It is based on a collinear factorization theorem at non-leading twist that relates the single-spin cross section to convolutions of twist-three quark-gluon correlation functions for the polarized proton with the usual parton distributions for the unpolarized proton and the pion fragmentation functions, and with hard-scattering functions calculated from an interference of two partonic scattering amplitudes: one with a two-parton initial state and the other with a three-parton initial state [7,8]. As was shown in [8], the phase needed to generate a single-spin asymmetry arises naturally in the hard-scattering functions, even at tree level, thanks to its pole structure. One finds the following expression for the spin-dependent cross section $[8,18]$ :

$E_{\ell} \frac{d^{3} \Delta \sigma\left(\vec{s}_{T}\right)}{d^{3} \ell} \propto \sum_{a, b, c} D_{c}^{h}(z) \otimes \phi_{b}\left(x^{\prime}\right) \otimes\left[T_{a, F}(x, x)-x\left(\frac{d}{d x} T_{a, F}(x, x)\right)\right] \otimes H_{a b \rightarrow c}(\hat{s}, \hat{t}, \hat{u})$

where $\hat{s}, \hat{t}, \hat{u}$ are the partonic Mandelstam variables, $\phi_{b}\left(x^{\prime}\right)$ and $D_{c}^{h}(z)$ are the usual unpolarized parton distribution and fragmentation functions, respectively, and the twistthree functions $T_{a, F}$ are defined as [8]

$$
T_{a, F}(x, x)=\int \frac{d y_{1}^{-} d y_{2}^{-}}{4 \pi} \mathrm{e}^{i x P^{+} y_{1}^{-}}\left\langle P, \vec{s}_{T}\left|\vec{\psi}_{a}(0) \gamma^{+}\left[\varepsilon^{s} T^{\sigma n \bar{n}} F_{\sigma}^{+}\left(y_{2}^{-}\right)\right] \psi_{a}\left(y_{1}^{-}\right)\right| P, \vec{s}_{T}\right\rangle .
$$



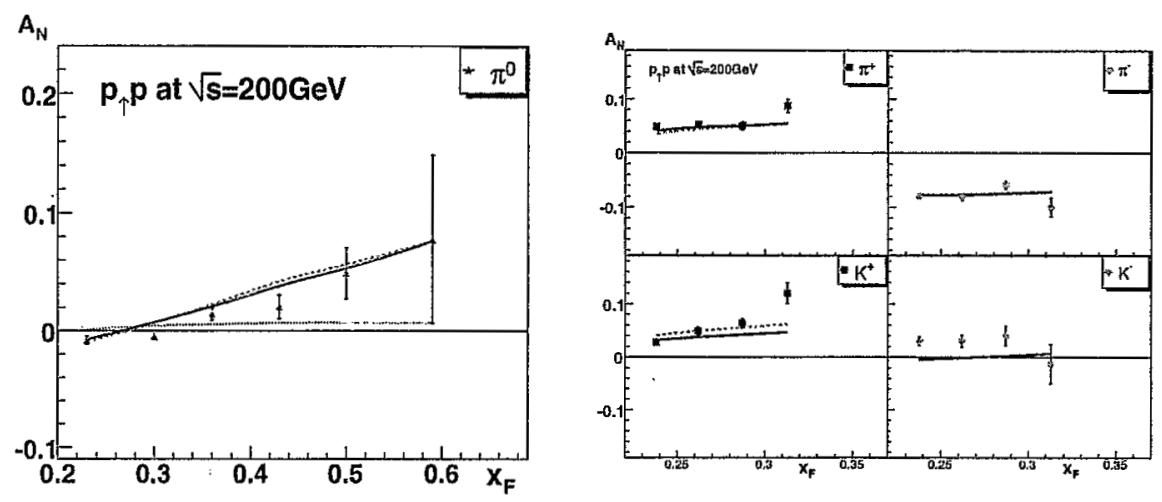

FIGURE 1. Comparison of the single-spin asymmetries $A_{N}$ using the two fit results of [18] to the preliminary RHIC data by the STAR [3] (left) and BRAHMS [5] (right) collaborations. The lower dotted line in the figure shows the contribution to $A_{N}$ by the "non-derivative" terms alone.

The sum in Eq. (2) runs over all flavors, with $H_{a b \rightarrow c}$ the associated hard-scattering functions. The $H_{a b \rightarrow c}$ are power-suppressed with respect to their unpolarized counterparts, and they are also down by $\sqrt{\alpha_{s}}$ due to the additional gluon that couples in the diagrams. As one can see, a specific combination of the $T_{a, F}$ occurs in Eq. (1), involving the derivative of $T_{a, F}$. Such derivative terms arise in the collinear expansion [8]. The terms in Eq. (1) without a derivative were only recently derived in [18].

Based on Eq. (1), one can perform phenomenology for the single-spin asymmetry, examining the salient features of the new RHIC data and of the earlier E704 fixedtarget pion production data. In [18] a simple model ansatz for the twist-three quarkgluon correlation functions $T_{a, F}(x, x)(a=u, \bar{u}, d, \bar{d}, s, \bar{s})$ was made, relating them to their unpolarized leading-twist counterparts. Figure 1 compares the fit results to the RHIC data. With new precise experimental information expected to arrive from RHIC we will be entering an era where detailed global analyses of the data on $A_{N}$ will become possible.

\section{STSA IN TWO-SCALE SITUATIONS}

Particularly interesting single-spin phenomena may occur when one has observables characterized by two very separate momentum scales, a hard scale $Q$, and a much lower measured transverse momentum $q_{\perp}$. As mentioned above, for such observables one may have a factorization in terms of TMD functions, for example:

$$
\hat{f}_{a}\left(x, k_{\perp}, s_{T}\right)=f_{a}\left(x, k_{\perp}\right)+\frac{1}{2} \Delta^{N} f_{a}\left(x, k_{\perp}\right) \frac{s_{T} \cdot\left(P \times k_{\perp}\right)}{\left|s_{T}\right||P|\left|k_{\perp}\right|},
$$

where the function $f_{a}\left(x, k_{\perp}\right)$ with $k_{\perp}=\left|k_{\perp}\right|$ is the unpolarized TMD parton distribution, and $\Delta^{N} f_{a}$ denotes the Sivers function [6].

For the Sivers function to exist, final/initial-state interactions are required, as well as an interference between different helicity Fock states of the nucleon. In the absence of interactions, the Sivers function would vanish by time-reversal invariance of QCD, hence 
it is often referred to as a "naively time-reversal-odd" (T-odd) distribution. As was shown in [14], the interactions are represented in a natural way by the gauge link that is required for a gauge-invariant definition of a TMD parton distribution. A particularly interesting aspect is that this makes the Sivers functions "non-universal" in the usual sense, i.e., they are not the same in each hard-scattering process. The non-universality has in fact a clear physical origin, and its closer investigation has turned out to be an extremely important and productive development in QCD. The gauge-link may be viewed as a rescattering of the parton in the color field of the nucleon remnant. Depending on the process, the associated color Lorentz forces will act in different ways on the parton. In DIS, the final-state interaction between the struck parton and the nucleon remnant is attractive. In contrast, for the Drell-Yan process it is repulsive. Therefore, the Sivers functions contribute with opposite signs to the single-spin asymmetries for these two processes [14]. This remarkable prediction really tests all concepts for analyzing hardscattering reactions that we know of, and awaits experimental testing.

The process-dependence of the Sivers functions will also manifest itself in more complicated QCD hard-scattering. An example is the single-spin asymmetry in dijet angular correlations $[19,20]$. As discussed above, the Sivers function represents a correlation of the form $s_{T} \cdot\left(P \times k_{\perp}\right)$ between the transverse proton polarization vector, its momentum, and the transverse momentum of the parton relative to the proton direction. A "left-right" imbalance in $k_{\perp}$ of the parton as expressed by the Sivers functions will affect the $\Delta \phi$ distribution of jets nearly opposite to the first jet and give the cross section an asymmetric piece around $\Delta \phi=\pi$. Figure 2 shows some model predictions for the single-spin asymmetry for this case [20]. Tremendous progress has been made recently in the understanding of the gauge links for this observable [16]. The more involved color structure of the hard-scattering functions has profound consequences on the gauge links. As a result, the Sivers functions for this reaction differ from those in DIS by more than just a sign. However, just like the relative sign between DIS and the Drell-Yan process, the process-dependence is calculable in QCD. The results shown in Figure 2 correctly take into account the process-dependence of the Sivers functions, to lowest order. Experimental verification would be major success for QCD theory. First, preliminary, data have been reported by the STAR collaboration at this conference [21].

The Collins functions [22] are another set of T-odd functions. They are TMD fragmentation functions and represent a correlation between the transverse spin of a fragmenting quark and the transverse momentum of the hadron relative to the "jet axis". Recently results for measurements in $e^{+} e^{-}$annihilation to two hadrons have been reported, which give direct evidence for the Collins effect [23]. The Collins functions are hoped to ultimately become tools for precise measurements of transversity $[2,24]$.

\section{RELATION BETWEEN MECHANISMS FOR STSA}

From what we discussed so far, the two mechanisms for single-spin asymmetries, twistthree quark-gluon correlation functions on the one hand and TMD distributions on the other, might appear to be essentially unrelated. However, a consistent theoretical description of the STSA for a hard process over its full kinematical regime requires both mechanisms to be present and to contain the same physics in the region where they 


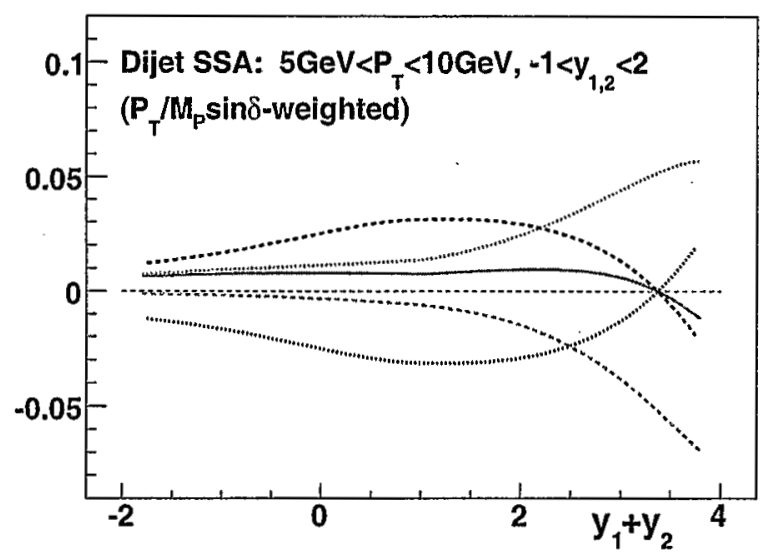

FIGURE 2. Sivers asymmetries for di-jet correlations at RHIC. The solid line is the prediction that takes into account the process dependence of the Sivers functions, while the two dashed black lines would be the corresponding predictions if the Sivers functions in di-jet production were identical to their counterparts in DIS or in the Drell-Yan process, respectively. For details, see [20].

both apply. As an example, we consider the STSA for the Drell-Yan process when the invariant mass $Q$ of the pair as well as its transverse momentum $q_{\perp}$ are measured [17].

At relatively large pair transverse momentum, $q_{\perp} \sim Q$, there is only one large scale, and the STSA will be power-suppressed in that scale. This directs us to use the ETQS mechanism with its collinear factorization involving the twist-three quark-gluon correlation functions and corresponding hard-scattering functions calculated at lowest order in perturbation theory from partonic $3 \rightarrow 2$ processes, as described in section 2 .

We can next investigate what happens in this case when we make the ratio $q_{\perp} / Q$ small, keeping however both scales perturbative, $Q \gg q_{\perp} \gg \Lambda_{\mathrm{OCD}}$. The ETQS mechanism will still apply here. At the same time, however, the factorization in terms of TMD distributions applies now [12], which involves the Sivers functions. If both mechanisms are internally consistent, they must describe the same physics in this region. In recent publications [17], we have demonstrated that the two mechanisms indeed provide the same description of the single-spin asymmetry for the Drell-Yan process in the regime $\Lambda_{\mathrm{QCD}} \ll q_{\perp} \ll Q$, and that there is a direct correspondence between the Sivers functions and the twist-three quark-gluon correlation functions. A smooth transition from the ETQS mechanism to the one based on TMD factorization occurs. A cartoon of this connection between the two mechanisms is given in Fig. 3. This unified picture should prove to be the best approach to phenomenological studies of single-spin asymmetries.

\section{ACKNOWLEDGMENTS}

I am grateful to D. Boer, C. Bomhof, X. Ji, C. Kouvaris, P. Mulders, J.W. Qiu, and F. Yuan for collaboration and discussions on some of the topics presented here. I benefited from many helpful and interesting discussions with F. Yuan. Supported by the U.S. Department of Energy under contract number DE-AC02-98CH10886. 


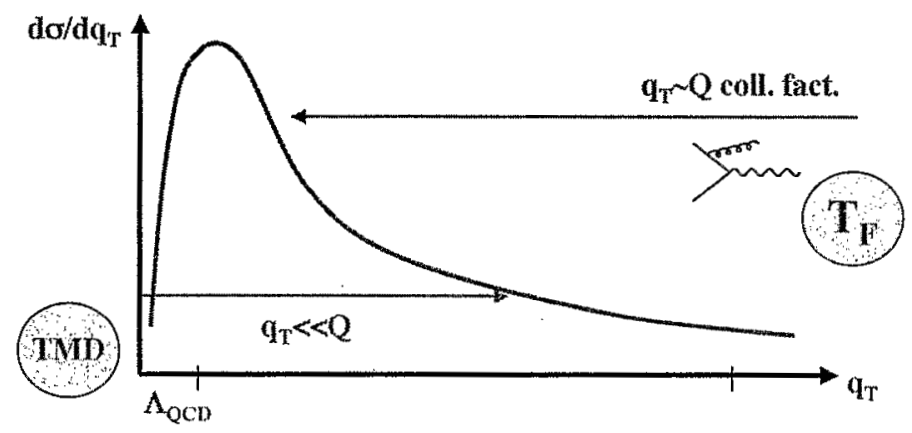

FIGURE 3. Cartoon of different kinematic regions $q_{T} \sim Q$ and $q_{\perp} \gg \Lambda_{\mathrm{QCD}}$ relevant for the singlespin asymmetry in the Drell-Yan process. In the region of overlap, $Q \gg q_{\perp} \gg \Lambda_{\mathrm{QCD}}$, both mechanisms describe the same physics [17].

\section{REFERENCES}

1. D.L. Adams et al. [E581 and E704 Collab.], Phys. Lett. B261, 201 (1991); D.L. Adams et al. [FNALE704 Collab.], Phys. Lett. B264, 462 (1991); K. Krueger et al., Phys. Lett. B459, 412 (1999).

2. A. Bravar [SMC], Nucl. Phys. A666, 314 (2000); A. Airapetian et al. [HERMES Collab.], Phys. Rev. Lett. 84, 4047 (2000); Phys. Rev. D64, 097101 (2001); Phys. Rev. Lett. 94, 012002 (2005); M. Diefenthaler, these proceedings; V.Y. Alexakhin et al. [COMPASS Collab.] Phys. Rev. Lett. 94, 202002 (2005); F. Bradamante, these proceedings.

3. J. Adams et al. [STAR Collab.], Phys. Rev. Lett. 92, 171801 (2004); L. Nogach, these proceedings.

4. S.S. Adler [PHENIX Collab.] Phys. Rev. Lett. 95, 202001 (2005); M. Chiu, these proceedings.

5. F. Videbaek [BRAHMS Collaboration], AIP Conf. Proc. 792, 993 (2005); J. H. Lee, these proc.

6. D.W. Sivers Phys. Rev. D41, 83 (1990); Phys. Rev. D43, 261 (1991).

7. E.V. Efremov, O.V. Teryaev, Sov. J. Nucl. Phys. 36, 140 (1982); Phys. Lett. B150, 383 (1985).

8. J.W. Qiu, G. Sterman, Phys. Rev. Lett. 67, 2264 (1991); Nucl. Phys. B378, 52 (1992); Phys. Rev. D59, 014004 (1999).

9. Y. Kanazawa, Y. Koike, Phys. Lett. B478, 121 (2000); Phys. Rev. D64, 034019 (2001).

10. M. Anselmino, F. Murgia, Phys. Lett. B442, 470 (1998); U. D'Alesio, F. Murgia, Phys. Rev. D70, 074009 (2004); M. Anselmino et al., Phys. Rev. D74, 094011 (2006).

11. P.J. Mulders, R.D. Tangerman, Nucl. Phys. B461, 197 (1996); [E:B484, 538 (1997)]; D. Boer, P.J. Mulders, Phys. Rev. D57, 5780 (1998); A. Bacchetta et al., hep-ph/0611265.

12. X. Ji, J.P. Ma, F. Yuan, Phys. Rev. D71, 034005 (2005); Phys. Lett. B597, 299 (2004).

13. J.C. Collins, A. Metz, Phys. Rev. Lett. 93, 252001 (2004).

14. S.J. Brodsky, D.S. Hwang, I. Schmidt, Phys. Lett. B530, 99 (2002); J.C. Collins, Phys. Lett. B536, 43 (2002); A.V. Belitsky, X. Ji, F. Yuan, Nucl. Phys. B656, 165 (2003).

15. D. Boer, P.J. Mulders, F. Pijlman, Nucl. Phys. B667, 201 (2003).

16. C.J. Bomhof, P.J. Mulders, F. Pijlman, Phys. Lett. B596, 277 (2004); Eur. Phys. J. C47, 147 (2006); A. Bacchetta et al., Phys. Rev. D72, 034030 (2005); C.J. Bomhof, P.J. Mulders, hep-ph/0609206.

17. X. Ji, J.W. Qiu, W. Vogelsang, F. Yuan, Phys. Rev. Lett. 97, 082002 (2006); Phys. Rev. D73, 094017 (2006); Phys. Lett. B638, 178 (2006).

18. C. Kouvaris, J.W. Qiu, W. Vogelsang, F. Yuan, hep-ph/0609238 (to appear in Phys. Rev. D).

19. D. Boer, W. Vogelsang, Phys. Rev. D69, 094025 (2004); W. Vogelsang, F. Yuan, Phys. Rev. D72, 054028 (2005).

20. C.J. Bomhof, P.J. Mulders, W. Vogelsang, F. Yuan, in preparation.

21. J. Balewski [STAR Collab.], these proceedings.

22. J.C. Collins, Nucl. Phys. B396, 161 (1993).

23. K. Abe et al. [Belle Collab.], Phys. Rev. Lett. 96, 232002 (2006); R. Seidl, these proceedings.

24. A. V. Efremov, K. Goeke, P. Schweitzer, Phys. Rev. D 73, 094025 (2006); A. Prokudin, these proceedings. 